\title{
Automation in the bioanalytical laboratory: what is the future?
}

\author{
"In the long journey to the hand-held bioanalytical laboratory, a more realistic milestone for this generation will be \\ the bioanalytical laboratory in a box."
}

Keywords: automation $\approx$ bioanalytical $=$ liquid-handling robots $\approx$ technology integration $\approx$ wet chemistry

A general trend in the advancement of analytical instrumentation is the improvement of functionalities and the simultaneous shrinking in physical size. Following this trend, the ultimate bioanalytical laboratory, as seen in many science fiction films, would be a highly integrated and automated hand-held device that can achieve non-invasive, instant readout of chemical concentrations in the body.

\section{Bioanalytical laboratory in a box}

In the long journey to the hand-held bioanalytical laboratory, a more realistic milestone for this generation will be the bioanalytical laboratory in a box (BLIB) [1]. Initially, there could be separate BLIBs for large-molecule and small-molecule bioanalyses. Since bioanalytical laboratory work can be roughly divided into wet-chemistry work and quantitative instrumental analysis, each of the BLIB's two major logical building blocks would be 'bioanalytical wet chemistry in a box' (BWCIB) and 'quantitative analysis in a box'. For quantitative instrumental analyses, over the past few decades, spectroscopy-based and MS-based detectors, as well as chromatography instrumentations, have seen leaps and bounds of progress in terms of functional improvement, extent of automation and hardware size reduction. That trend is continuing [2], so the 'quantitative analysis in a box' part of BLIB is already within reach. What is lagging way behind is the BWCIB part, to which an important prelude would be the 'bioanalytical wet chemistry kiosk' (BWCK) [3].

\section{Bioanalytical wet-chemistry kiosk}

The idea here is linear: first automate the vast majority of bioanalytical wet-chemistry work (sample analyses and assay development) and condense them into a kiosk; then gradually improve it, raise the degree of integration, and shrink its size further to BWCIB through technological breakthroughs, such as microfluidics and/or nanotechnology. Depending on the pace of maturation of microsampling, microfluidics applications and nanotechnology in the bioanalytical laboratories [4], the BWCK development work may even result in BWCIB directly. Even if that does not turn out to be the case, the development of BWCK will be an important stepping stone for BWCIB.

The late inception of BWCK is probably because we are still in the relatively early stage of wet chemistry automation. Microtiter plate, the foundation of wet chemistry automation, was invented in the 1950s. But it only started to become widely used in high-throughput screening laboratories in the mid-1990s. Bioanalytical applications started even later. Industrial standard was nonexistent until 2003, when the American National Standards Institute published a series of microtiter plate-related standards. The flourishing of liquid-handling robots in the life-science laboratories followed. Robot manufacturers naturally want their products to be versatile, hence the current array of generalpurpose robots, each with their independent, different but self-sufficient proprietary scripting environment. At the bioanalytical user end, since making good use of such general-purpose robots for bioanalytical workflows requires at least some scripting skills, and not many bioanalysts know how to script, the adoption has been slow [5]. The perception that the entire bioanalytical wet-chemistry workflow seems so chaotic and could go in any direction does not help either. The lack of scripting skills combined with the seemingly 'chaotic and unpredictable' nature of bioanalytical wet-chemistry work is probably why many laboratories currently do piecemeal automation - program the robot to do single or very few automation steps at a time.

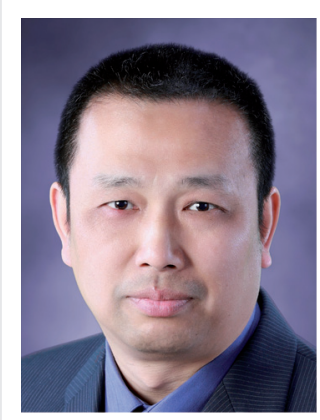

Ming Li

Biogen Idec, I4 Cambridge Center, Cambridge, MA 02142, USA

E-mail: ming.li@biogenidec.com 
In the computer science field, there is this consensus that if a process can be described by a finite number of variables, in a finite number of steps, then no matter how complex the process seems, it can be automated. Recently published studies [6-8] demonstrate that the majority of seemingly chaotic and unpredictable small-molecule bioanalytical wet-chemistry workflows, be they routine sample analyses or assay development, is this kind of 'complex process', and so can be encapsulated into computer logic and automated, on a single robotic platform. Large molecules will be similar [9]. While unleashing the full potential of the current robots for bioanalyses, these studies also revealed the limitations of the current robots and what bioanalysts want in terms of hardware capabilities and software functionalities. They are essentially early proof-of-concept work for the BWCK. Much additional developmental work is needed to make BWCK a reality. Since it is going to be a hardware tool, and the bioanalytical industry is not in the tool-making business, the development work needs to be done by the robot companies. Hardware wise, accessories such as vortex, centrifuge, capper, de-capper, washer, incubator, plate reader, and so on, needs to be seamlessly integrated. 'Seamlessly' here means it cannot be simply throwing a retail piece of hardware accessory, for example, a vortex, onto the existing deck. Robot deck layout, plate transport mechanism and component inter-connections need to be redesigned. Software wise, the scripting layer needs to be eliminated, bioanalytical user interface, bioanalytical business logic, liquid-handling control, peripheral drivers, data connection to enterprise laboratory information management system, electronic laboratory environment, messaging servers and so on all need to be united under one roof. It would take brilliant mechanical, electrical and computer engineers some time to do it, but this is a case of integrating existing technologies, not scientific or technological breakthrough. Therefore, if there is a will, there will be a way. The problem is robot manufacturers may not have the will.

\section{Automation development collaboration}

By commercial instinct, robot companies want to diversify and make their robots as versatile as possible, penetrating as many market sectors as possible. Betting on one particular market sector is counter-intuitive and is a significant strategic business risk. Therefore, even though BWCK's benefits are fairly obvious to bioanalysts and its potential impact on drug-development timelines and costing is huge, the robot manufacturers may not necessarily be willing to do it. Even if they are willing, they may not have the bioanalytical expertise to do it properly. Therefore, it is up to the bioanalytical industry to influence robot companies' marketing and product-development teams, weighing the bioanalytical market sector size, potential implications for other life-science laboratory workflows, potential profits against product $\mathrm{R} \& \mathrm{D}$ costs, risks and so on, and collaborate with them technically to make BWCK or even BWCIB a reality, and constantly improve it.

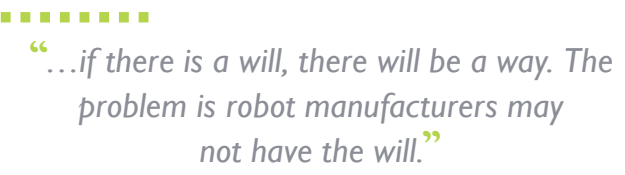

Before BWCK materializes, is there anything else the bioanalytical industry can do? Yes, there is. Now that the Simple to use; Mindful of user errors; Assay class automation; Robust, rugged and reliable; Tied into organization's IT systems (SMART) way of bioanalytical wet-chemistry automation on existing robotic platforms [3] is public knowledge [7,8], ironically, developing process-improving, productivity-boosting SMART automation solutions for all bioanalytical assay types and assay development work has become a production process in itself, which could use some productivity boost as well. For example, my team, being SMART automation practitioners, have found ourselves not being able to keep up with the development demand. It is probably more or less the same in other organizations, since the resource allocated to capability and process improvement is limited within each life-science organization. Therefore, automation development resource sharing seems to be in order. The justification, feasibility, format and implementation details of current bioanalytical automation development collaboration could be the topics of separate discussions. My preliminary conclusion is: it benefits everyone, including bioanalytical bench scientists.

\section{The human consideration}

Some of my bioanalytical bench scientists colleagues asked me: "if you automate everything, doesn't that mean we will all lose our jobs, including yours?" My answer is no. Most likely, the resource savings from BWCK, BWCIB or 
BLIB will be shifted elsewhere; for example, upstream to in-life portions of PK studies. In the above example, more PK studies will arrive and each bioanalyst will just be expected to do more. There will always be sparing laboratory work that is not automated. Cognitive work such as experimental design and data interpretation cannot be easily automated. If a bioanalyst is presented with two future career paths: one filled with massive manual pipetting, one filled with operation of BWCK, BWCIB or BLIB and intensive scientific cerebration, I think the choice will be obvious. As for my fellow laboratory automation scientists and technology enthusiasts, there will be plenty of things to work on after the current wave of SMART automation work is done. If you are a gadget person, you have microsampling, microfluidics and lab-ona-chip to toy with. If software is your cup of tea, you have data mining, pattern recognition and artificial intelligence to ponder [10,11]. The work for bioanalysts and laboratory automation scientists never ends.

\section{Conclusion}

For the immediate future, the bioanalytical industry could either collectively collaborate with robot manufacturers on their product development, collaborate within the industry on bioanalytical wet-chemistry automation development, or just stay in the current course and do more or less the same automation development work in separate silos. Our future is really what we make of it. However, any way I look at it, the future bioanalytical laboratory will be highly automated.

\section{Financial \& competing interests disclosure}

The author has no relevant affliations or financial involvement with any organization or entity with a financial interest in or financial conflict with the subject matter or materials discussed in the manuscript. This includes employment, consultancies, honoraria, stock ownership or options, expert testimony, grants or patents received or pending, or royalties.

No writing assistance was utilized in the production of this manuscript.

\section{References}

1 Singleton C, Li M. Automated sample preparation method development. In: Eliminating Bottlenecks for Efficient Bioanalysis: Practices and Applications in Drug Discovery and Development. Future Science Group, London, UK (2013) (In Press).

2 Reisch M. Tiny tools: instrument makers pack increased power into miniaturized analytical gear. Chem. Eng. News 91(34), 11-15 (2013).

3 Allinson JL, Blick KE, Cohen L, Higton D, Li M. Ask the experts: automation: part I. Bioanalysis 5(16), 1953-1962 (2013).

4 Yuan L, Ji QC. Automation in new frontiers of bioanalysis: a key for quality and efficiency. Bioanalysis 4(23), 2759-2762 (2012).

5 Cohen LH. Surrendering to the robot army: why we resist automation in drug discovery and development. Bioanalysis 4(9), 985-987 (2012).
6 Tweed J, Gu Z, Xu H et al. Automated sample preparation for regulated bioanalysis: an integrated multiple assay extraction platform using robotic liquid handling. Bioanalysis 2(6), 1023-1040 (2010).

7 Li M, Chou J, Jing J et al. MARS: bringing the automation of small-molecule bioanalytical sample preparations to a new frontier. Bioanalysis 4(11), 1311-1326 (2012).

8 Li M, Chou J, King K, Yang L. ASPECTS: an automation-assisted SPE method development system. Bioanalysis 5(13), 1661-1676 (2013).

Patel V, Leach D, Hornberger M et al. Automating bioanalytical sample analysis through enhanced system integration. Bioanalysis 5(13), 1649-1659 (2013).

10 Zelesky V, Schneider R, Janiszewski J, Zamora I, Ferguson J, Troutman M. Software automation tools for increased throughput metabolic soft-spot identification in early drug discovery. Bioanalysis 5(10), 1165-1179 (2013).

11 Shou WZ, Zhang J. Recent development in software and automation tools for highthroughput discovery bioanalysis. Bioanalysis 4(9), 1097-1109 (2012). 